\title{
Evaluation of different diagnostic methods of bacterial vaginosis
}

\author{
Gamal F. M. Gad'1 , Ahmed R. El-Adawy², Mohammed S. Mohammed ${ }^{3}$, \\ Abobakr F. Ahmed ${ }^{1}$, Heba A. Mohamed ${ }^{1}$ \\ ${ }^{1}$ Microbiology department, Faculty of Pharmacy, Minia University \\ ${ }^{2}$ Gynecology and Obstetrics department, Faculty of Medicine, Minia University \\ ${ }^{3}$ Microbiology department, Faculty of Medicine, Minia University
}

\begin{abstract}
Bacterial vaginosis (BV) is an ecological disorder of the vaginal microbiota that affects millions of women annually, and is associated with numerous adverse health outcomes including pre-term birth and the acquisition of sexually transmitted infections. This study aimed to determine the incidence rate of bacterial vaginosis among women and examine the effect of some risk factors and demographic features on $B V$ incidence. Also aimed to compare between the different methods of $B V$ diagnosis. The results revealed that from the 100 patients, $33 \%$ were diagnosed as bacterial vaginosis, $23 \%$ were having candidiasis, $8 \%$ aerobic vaginitis, 3\% trichomoniasis and 33\% were normal. The highest number of BV positive cases was in the age group of (26-35)Yrs, 94\% of the 33 BV positive cases were using vaginal douches and 94\% were married. BV positive cases using IUD were equal to $B V$ positive cases using hormonal contraceptives including injection and tablets $(12 / 33,36.4 \%)$. BV was diagnosed in $33 \%, 38 \%, 36 \%, 30 \%$ and $34 \%$ of patients using Gram stain, culture, Amsel criteria, BV Blue test ${ }^{\circledR}$ and $q P C R$ respectively. When the different diagnostic methods were compared to Gram stain as a gold standard, qPCR had the highest sensitivity, accuracy and negative predictive value while BV Blue test had the highest specificity and positive predictive value.
\end{abstract}

Key words: Bacterial vaginosis, Nugent score, Amsel criteria, BVBlue test.

\section{Introduction}

Bacterial vaginosis (BV) is a polymicrobial syndrome with acquisition of several fastidious bacteria, concurrent with a decrease of lactobacilli, the dominant constituents in normal vaginal flora [1]. It is a condition characterized by replacement of vaginal lactobacilli with predominantly anaerobic micro-organisms such as Gardnerella vaginalis, Prevotella, Peptostreptococcus and Bacteroides spp. [2]. Although BV is often asymptomatic, it still is, along with vulvovaginal candidiasis, the most common cause of vaginitis, and hence among the commonest reasons for women to seek medical help [3]. BV is common in low social economic groups where the reported incidence is 20-49\%. Its reported rate is $45-55 \%$ in African American, $20-30 \%$ in Asian women and 5-15\% in Caucasian women [4].

There is an evidence that BV, whether it is asymptomatic or symptomatic, is an independent risk factor for severe reproductive tract and obstetric sequelae, including pre-term delivery, pre-term labor, low birth weight, post abortion endometritis, post-partum endometritis [5], development of Pelvic inflammatory disease (PID) as reported by [6], acquisition of sexually transmitted diseases (STIs) as proved by [7], acquisition and transmission of HIV [8]. Due to these serious complications, there has been an increase of interest in bacterial vaginosis during the last decade, regarding its diagnosis and treatment [9].

The diagnosis for bacterial vaginosis remained difficult and controversial due to lack of diagnostic tools. Clinically, BV can be identified by the presence of at least three of the four Amsel criteria: an elevated vaginal $\mathrm{PH}$, an increased vaginal discharge, the presence of clue cells and an amine odor after the addition of potassium hydroxide [10]. Another method used to detect BV is Gram staining of vaginal smears and evaluation of them according to Nugent scoring system which scores vaginal smaers from 0 to 10 depending on the numbers of three bacterial morphotypes observed on the slide [11,12].

The culture- based microbial detection method of the vaginal fluid is performed for the detection of the causative agents of intravaginal infections including bacterial vaginosis. In women with BV, Gardnerella vaginalis is almost universally present (99\%) and majority have Atopobium vaginae (96\%) infection [13], Culture of these two bacteria will be useful as a diagnostic tool of BV.

The BV Blue Kit is a bedside rapid test kit to diagnose bacterial vaginosis. It involved the detection of vaginal fluid sialidase activity which is produced by the organism causing bacterial vaginosis. BV Blue detects vaginal fluid sialidase activity at levels of $\geq 7.8 \mathrm{U}$ [14].

PCR-based tests are being used for molecular diagnosis of BV, mostly based upon molecular quantification of G. vaginalis and A. vaginae [15]. These two species co-occur in the large majority of BV patients, and the possibility of combining the detection of these two species for an accurate diagnosis of BV has been suggested [16, 17]. 
This study aimed to monitor the prevalence of BV among women complaining from vaginitis and excessive vaginal discharge and evaluation of the different commonly used methods of $\mathrm{BV}$ diagnosis including Amsel criteria, culture, BVBlue test ${ }^{\circledR}$ and quantitative real time PCR, regarding their sensitivity, specificity, positive predictive value, negative predictive value and accuracy using Gram stain with Nugent score as a gold standard.

\section{Material and Methods}

2.1. Patients: The study was carried out on one hundred patients attending family planning clinic at Minia University hospital for obstetrics and gynecology, Minia, Egypt during the period between June 2012 to June 2013. A total of 500 vaginal swabs (five swabs for each patient) and 100 vaginal lavage were taken from 100 patients complaining from symptoms of vaginitis or excessive vaginal discharge. The patients included in this study were in the age range from (18-45) years.

Patients were subjected to questionnaire including demographic data like age, marital status and pregnancy. They were asked about their presenting complaints, contraceptive use, menstrual history, previous episodes of vaginal infections, last sexual intercourse, medical history, history of using vaginal douches or any vaginal preparations and history of taking any antimicrobial agents either systemic or vaginal. All patients were examined using speculum without lubrication and the presence of vaginal erythema and vaginal discharge was recorded with description of the color, consistency and odor of discharge. Then vaginal swabs and lavages were taken and transported immediately to the laboratory either to the microbiology unit at Minia university hospital or to the microbiology laboratory at faculty of pharmacy, Minia University.

2.2. Ethics statement: Ethical approval to perform the study was obtained from the institutional review board of Minia University. Written informed consents were obtained from all patients recruited in this study.

2.3. Sample processing: Five swabs were taken from each patient; one was collected from the lower one third of the vaginal wall and used for BVBlue ${ }^{\circledR}$ test immediately at the time of sample collection. The other four swabs were taken from the lateral walls or the posterior fornix of the vagina, one is used for wet mount examination, another one was used for $\mathrm{pH}$ measurement and whiff test, another one rolled on a slide and used for gram staining and the forth swab is embedded in Amies transport medium and then used for culture. Cervicovaginal lavage (CVL) was collected from each patient by insertion of nonlubricated speculum into the vaginal vault, then the vagina is irrigated with $10 \mathrm{ml}$ of sterile saline followed by aspiration from the posterior fornix. CVL was held on ice until being processed within 6 hours of collection. CVL was gently vortexed to evenly distribute cells before aliquoting and freezing at $-80^{\circ} \mathrm{C}$.

\subsection{Amsel criteria [10]:}

Clinical diagnosis using Amsel criteria requires that at least three of the following four criteria are met: first, a vaginal $\mathrm{pH}$ of greater than $\mathrm{pH} 4.5$; second, the presence of clue cells in the vaginal fluid when examined in a wet mount; third, a milky homogeneous vaginal discharge (There must not be any granular elements; the fluid must be completely homogenous); and finally, positive Whiff (Sniff) test. Vaginal pH was measured by rolling the swab over a pH strip immediately after swabbing and the resulted color is translated into the $\mathrm{pH}$ value. Clue cells are epithelial cell studded with Gram variable coccobacilli, it was detected by microscopic examination of wet mount which was made by mixing the vaginal discharge from the swab with a drop of saline. A homogenous milky discharge was detected by examination after speculation. Whiff test was done by mixing the vaginal discharge from a swab with a drop of $10 \% \mathrm{KOH}$; a positive result is a "fishy" amine odor. Wet mount was used also for detection of trichomonads.

\subsection{Gram stain and Nugent scoring system [11]:}

After rolling the vaginal swab on a microscopic slide, it was left to dry then gram stained with gram staining protocol, then it was read by a single experienced microbiologist to eliminate the possibility of interobserver difference. Slides were read according to Nugent score as follows: Morphotypes were counted as the average number of bacteria in 10-20 oil immersion field. The Nugent score was calculated by assessing for the presence of large gram-positive rods (Lactobacillus morphotypes; decrease in Lactobacillus scored as 0 to 4), small gram-variable and gram-negative rods ( $G$. vaginalis and Bacteroides morphotypes; scored as 0 to 4), and curved gram-variable rods (Mobiluncus spp. morphotypes; scored as 0 to 2), After the amount of each morphotype detected on the smear was graded it was then allocated a score as shown in Table (1). Then total score calculated from 0 to 10. A score of 1-3, considered normal, a score of 4-6 considered intermediate (means an intermediate state between normal and BV) and a score of 7 to 10 was consistent with BV. This method is considered the gold standard for diagnosis of BV. 
Gram stained slides were examined also for candida spp. in both spherical or pseudohyphae form for diagnosis of candidiasis and examined for polymorphnuclear cells (PMNs) count, parabasal cells and gram positive cocci for diagnosis of aerobic vaginitis.

\subsection{Culture:}

Swabs for culture were embedded in Amies transport medium, and then transported immediately to the laboratory. Swabs were then cultured by semi quantitative culture method and a score of $2+$ or more was considered positive. For isolation of Gardnerella vaginalis, swabs were streaked on a selective Human Blood Tween (HBT) bilayer agar medium [18] and incubated at $37^{\circ} \mathrm{C}$ for $48-72$ hours in a humid $5-10 \% \mathrm{CO} 2$ atmosphere. For isolation of Atopobium vaginae, swabs were streaked on trypticase soy agar (TSA) with 5\% and incubated anaerobically at $37^{\circ} \mathrm{C}$ for $24-48$ hours.

Table (1): Nugent Scoring system for Gram-stained vaginal smears

Score Lactobacillus Morphotype Gardnerella and Bacteroides Morphotype Curved Gram variable
rods

\begin{tabular}{lccc}
\hline 0 & $4+$ & 0 & 0 \\
1 & $3+$ & $1+$ & $1+$ or $2+$ \\
2 & $2+$ & $2+$ & $3+$ or $4+$ \\
3 & $1+$ & $3+$ & $4+$ \\
4 & 0 & $4+$ & $4+$
\end{tabular}

$0=$ no morphotype present, $1+=<1$ morphotype present, $2+=1$ to 4 morphotype present, $3+=5$ to 30 morphotype present, $4+=>30$ morphotype

2.6.1. Identification of G. vaginalis: G. vaginalis colonies on HBT agar appear as tiny greyish smooth, round and $\beta$-hemolytic colonies (on human blood not sheep blood). Gram stain showed either gram negative or gram variable bacilli or coccobacilli. G. vaginalis is negative oxidase and catalase [19].

2.6.2. Identification of Atopobium vaginae: Atopobium vaginae shows Greyish-white non-hemolytic colonies after $48 \mathrm{~h}$ culture on blood agar in anaerobic conditions. Gram staining shows gram-positive elliptical cocci-shaped bacteria as single cells, in pairs or short chains [20].

\subsection{BVBlue test ${ }^{\circledR}$ :}

The OSOM BVBLUE test ${ }^{\circledR}$ provided by Gryphus Diagnostics, L.L.C. is a chromogenic test done by immersing the swab into the tube containing IBX 4041 (chromogenic substrate), and incubated at $37{ }^{\circ} \mathrm{C}$ for 10 minutes and then, 1-2 drops of $\mathrm{BV}$ Blue developer solution $(\mathrm{NaOH}$ solution $40 \mathrm{mg} / \mathrm{ml})$ was added. Development of intense blue color within 3 minutes indicated the test as positive [21].

\subsection{Quantitative real time PCR ( $q P C R)$ [22]:}

2.8.1. DNA extraction: Standard serial dilutions of bacetria or cervicovaginal lavage (CVL) samples were centrifuged for $30 \mathrm{~min}$ at $13000 \mathrm{Xg}$. DNA was extracted from the pellets produced after centrifugation with QIAamp DNA Mini Kit (Qiagen, Germany) according to the manufacturer's protocol "Applied Biosystems, USA" automatically using qiacube.

2.8.2. Quantitaive real time PCR: Quantitative real time PCR was carried out using Fast $\mathrm{SYBR}{ }^{\circledR}$ Green Master Mix (Applied Biosystems, USA). It targeted Lactobacillus species, A. vaginae and G. vaginalis, Amplification was carried out out using a gene amp 5700 thermocycler (Applied Biosystems) with a thermocycle profile and the PCR condition for G. vaginalis and A. vaginae assays was $95^{\circ} \mathrm{C}$ for 2 min, followed by 40 cycles at $95^{\circ} \mathrm{C}$ for $1 \mathrm{~min}, 63^{\circ} \mathrm{C}$ (for G. vaginalis) and $65^{\circ} \mathrm{C}\left(\right.$ A. vaginae ) for $1 \mathrm{~min}$ and $72^{\circ} \mathrm{C}$ for $1 \mathrm{~min}$. For Lactobacillus sp the PCR condition was: $95^{\circ} \mathrm{C}$ for $10 \mathrm{~min}$, followed by 40 cycles at $95^{\circ} \mathrm{C}$ for $30 \mathrm{sec}$ and $60^{\circ} \mathrm{C}$ for $1 \mathrm{~min}$ (simultaneous annealing and extension). The final results were expressed as copies of microorganism DNA per $1 \mathrm{~mL}$ of vaginal suspension. In each thermocycler run, six standards consisting of $10^{2}$ to $10^{7}$ copies of DNA from the appropriate bacterium were included to generate a standard curve, from which concentrations of samples were obtained. Menard et al., [23] reported that a combination of A. vaginae and G. vaginalis analyzed quantitatively could be used to accurately differentiate BV-positive from BV-negative samples, using threshold concentrations of $10^{8}$ copies $/ \mathrm{ml}$ and $10^{9}$ copies $/ \mathrm{ml}$, respectively. Also, they reported a threshold of Lactobacillus concentration of $\geq 10^{8}$ copies $/ \mathrm{ml}$.

Primers:

Gardnerella vaginalis $\left(5^{\prime} \rightarrow 3\right.$ '): Forward (GGAAACGGGTGGTAATGCTGG). 
Reverse (CGAAGCCTAGGTGGGCCATT)

Atopobium vaginae $\left(5^{\prime} \rightarrow 3^{\prime}\right)$ : Forward (GTTAGGTCAGGAGTTAAATCTG).

Reverse (TCATGGCCCAGAAGACC).

Lactobacillus species $\left(5^{\prime} \rightarrow \mathbf{3}^{\prime}\right)$ : Forward (TACATCCCAACTCCAGAACG).

Reverse (AAGCAACAGTACCACGACC).

Standard strains \{supplied by the American Type Culture Collection (ATCC)\}:

Atopobium vaginae ATCC ${ }^{\circledR}$ BAA-55, Gardnerella vaginalis ATCC $^{\circledR} 14018$ and Lactobacillus crispatus ATCC $^{\circledR} 33820$, used to make standard concentrations.

2.9. Statistical analysis: Standard calculations for the measurement of sensitivity, specificity, positive predictive value (PPV), negative predictive value (NPV) and accuracy were used, considering gram stain and Nugent scoring as a gold standard. Statistical analysis was conducted using SPSS software (version 11). Kappa values and degrees of agreement of the diagnostic methods were determined.

\section{Results}

The results revealed that from the 100 patients, $33(33 \%)$ were diagnosed as bacterial vaginosis (by Nugent method), 23 (23\%) were having candidiasis ( 8 of them mixed with bacterial vaginosis), 8 (8\%) aerobic vaginitis, $3(3 \%)$ trichomoniasis and $33(33 \%)$ were normal. Relation of BV incidence with the demographic features and risk factors are described in Fig (1). It is observed that the highest number of BV positive cases were in the age group of (26-35)Yrs. All BV positive cases were non pregnant $(33 / 33,100 \%), 31(94 \%)$ of the $\mathrm{BV}$ positive cases were married and $31(94 \%)$ of the BV positive cases were using vaginal douches while only 2 $(6.1 \%)$ cases were not using vaginal douches. It is observed that the number of BV positive cases using IUD was equal to the number of BV positive cases using hormonal contraceptives including injection and tablets $(12 / 33$, $36.4 \%)$.
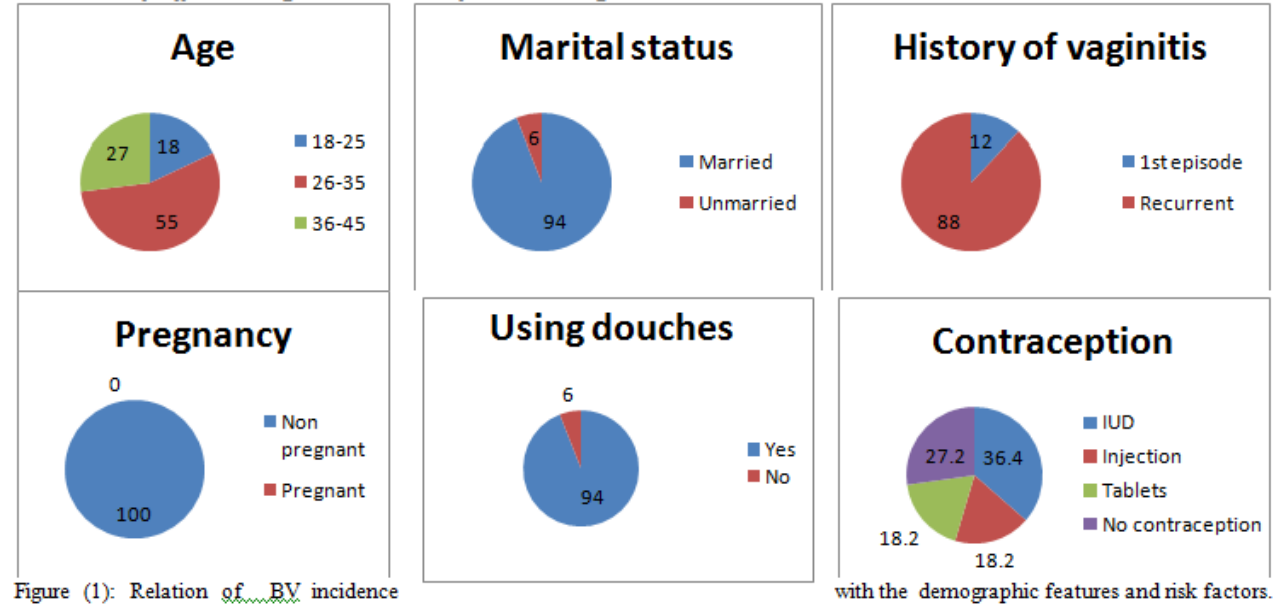

3.1. Nugent score: According to Nugent scoring system 33 (33\%) of patients were BV positive with Nugent score 7-10 and $67(67 \%)$ of patients were BV negative including $27(27 \%)$ patients with Nugent score 1-3 (normal flora) and 40 (40\%) patients with Nugent score 4-6 (intermediate flora).

3.2. Culture: Gardnerella vaginalis was isolated from $45(45 \%)$ patients, with growth on HBT agar varied from $2+$ to $4+$. Atopobium vaginae was isolated from 38 (38\%) patients, with growth on TSA with 5\% blood also ranged from $2+$ to $4+$. While the number of patients from whom both $G$. vaginalis and A. vaginae were recovered, was $38(38 \%)$. We considered positive for culture as a method of BV diagnosis if the case was positive for both G. vaginalis and A. vaginae culture. So according to culture, the number of BV positive cases is $38(38 \%)$ while the number of $\mathrm{BV}$ negative cases is $62(62 \%)$.

3.3. Amsel criteria: It was observed that $36(36 \%)$ of the 100 cases, were BV positive, they met three or four of Amsel criteria while 64 (64\%) cases were BV negative. Table (2) shows the results of Amsel criteria including presence of milky homogenous discharge, increase of $\mathrm{pH}(>4.5)$, positive whiff test and presence of clue cells in $\mathrm{BV}$ positive cases.

3.4. BVBlue test ${ }^{\circledR}$ : According to BVBlue test, $30(30 \%)$ patients were BV positive while $70(70 \%)$ patients were BV negative. Fig. (2) shows BVBlue positive and BVBlue negative results. 
Table (2): Incidence of Amsel criteria in BV positive cases

\begin{tabular}{|c|c|c|c|c|}
\hline \multirow{2}{*}{ Amsel criteria } & \multicolumn{2}{|c|}{ Positive cases } & \multicolumn{2}{c|}{ Negative cases } \\
\cline { 2 - 5 } & No. & $\%^{*}$ & 3 & No. \\
\hline 1- Homogenous discharge & 33 & 91.7 & 4 & 8.3 \\
\hline 2- $\mathrm{pH}>$ 4.5 & 32 & 88.9 & 2 & 11.1 \\
\hline 3- Whiff test & 34 & 94.4 & 2 & 5.6 \\
\hline 4- Presence of clue cells & 34 & 94.4 & 5.6 \\
\hline
\end{tabular}

* Percentage calculated in relation to the number of positive cases according to Amsel criteria (36).

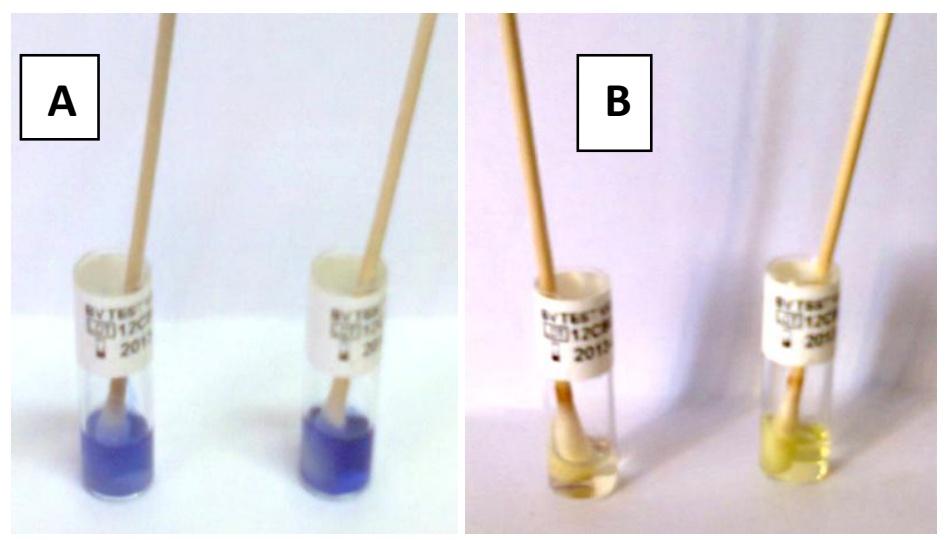

Figure (2): BVBlue test, Positive result appears as blue color (A) and negative result appears as yellow color $(B)$.

3.5. Quantitative real time PCR (qPCR): It was observed that $32(32 \%)$ cases contained G. vaginalis DNA level $\geq 10^{9}$ copies $/ \mathrm{mL}$ and $30(30 \%)$ cases contained $A$. vaginae DNA level $\geq 10^{8}$ copies $/ \mathrm{mL}$. The number of cases showing $G$. vaginalis DNA level $\geq 10^{9}$ copies $/ \mathrm{mL}$ and/or $A$. vaginae DNA level $\geq 10^{8}$ copies $/ \mathrm{mL}$ were $34(34 \%)$ cases. The combination of an A. vaginae DNA level $\geq 10^{8}$ copies $/ \mathrm{mL}$ and a G. vaginalis DNA level $\geq$ $10^{9}$ copies $/ \mathrm{mL}$ demonstrated the best predictive criteria for BV diagnosis. According to this, 34 (34\%) cases were BV positive while $66(66 \%)$ cases were BV negative. Regarding Lactobacillus spp. concentration, 62 $(62 \%)$ cases were having Lactobacillus spp. concentration $\geq 10^{8}$ copies $/ \mathrm{mL}$ while $38(38 \%)$ were having Lactobacillus spp. concentration $<10^{8}$ copies $/ \mathrm{mL}$. All the 32 cases having A. vaginae DNA level $\geq 10^{8}$ copies/mL and/or $G$. vaginalis DNA level $\geq 10^{9}$ copies $/ \mathrm{mL}$, had also Lactobacillus spp. concentration of < $10^{8}$ copies $/ \mathrm{mL}$.

\subsection{Comparison of different diagnostic methods with Nugent score:}

Values of true and false positive results and true and false negative results of the different diagnostic methods are mentioned in Table (3). Results of sensitivities, specificities, positive predictive values (PPV), Negative predictive values (NPV) and accuracies of the different diagnostic methods in comparison with Nugent score as a gold standard are mentioned in Table (4) and represented in Fig. (3).

It was observed that, qPCR had the highest sensitivity $(96.9 \%)$, accuracy $(97 \%)$ and negative predictive value $(98.5 \%)$ while BVBlue test had the highest specificity $(98.5 \%)$ and positive predictive value (96.6\%). Amsel criteria and BVBlue test had the least sensitivity (88\%), culture had the least specificity $(88 \%)$ and positive predictive value $(79 \%)$ and Amsel criteria had the least negative predictive value (94\%). Amsel criteria and culture had the least accuracy (89\%). When Kappa value " $\mathrm{K}$ " was calculated, it was found that Culture, Amsel criteria, BVBlue test \& qPCR had the following Kappa values, 0.76, 0.76, 0.78 \& 0.93 respectively. This means that culture, Amsel criteria \& BVBlue test had substantial agreement $(\mathrm{K}=0.61-0.80)$ with the gold standard and qPCR had almost perfect agreement with the gold standard $(\mathrm{K}>0.81)$.

Table (3): True and false results of the different diagnostic methods

\begin{tabular}{|l|c|c|c|c|}
\hline \multicolumn{1}{|c|}{ Methods } & True positive & False positive & True negative & False negative \\
\hline 1- Culture & 30 & 8 & 59 & 3 \\
\hline 2- Amsel criteria & 29 & 7 & 60 & 4 \\
\hline 3- BVBlue test & 29 & 1 & 66 & 4 \\
\hline 4- qPCR & 32 & 2 & 65 & 1 \\
\hline
\end{tabular}


Table (4): Sensitivity, Specificity, Accuracy, Positive predictive value (PPV), and Negative predictive value (NPV) of the different diagnostic methods

\begin{tabular}{|c|c|c|c|c|c|}
\hline Methods & Sensitivity & Specificity & Accuracy & PPV* & NPV*** \\
\hline 1- Culture & 90.9 & 88 & 89 & 79 & 95.2 \\
\hline 2- Amsel criteria & 88 & 89.6 & 89 & 81 & 94 \\
\hline 3- BVBlue test & 88 & 98.5 & 95 & 96.6 & 94.3 \\
\hline 4- qPCR & 96.9 & 97 & 97 & 94 & 98.5 \\
\hline
\end{tabular}

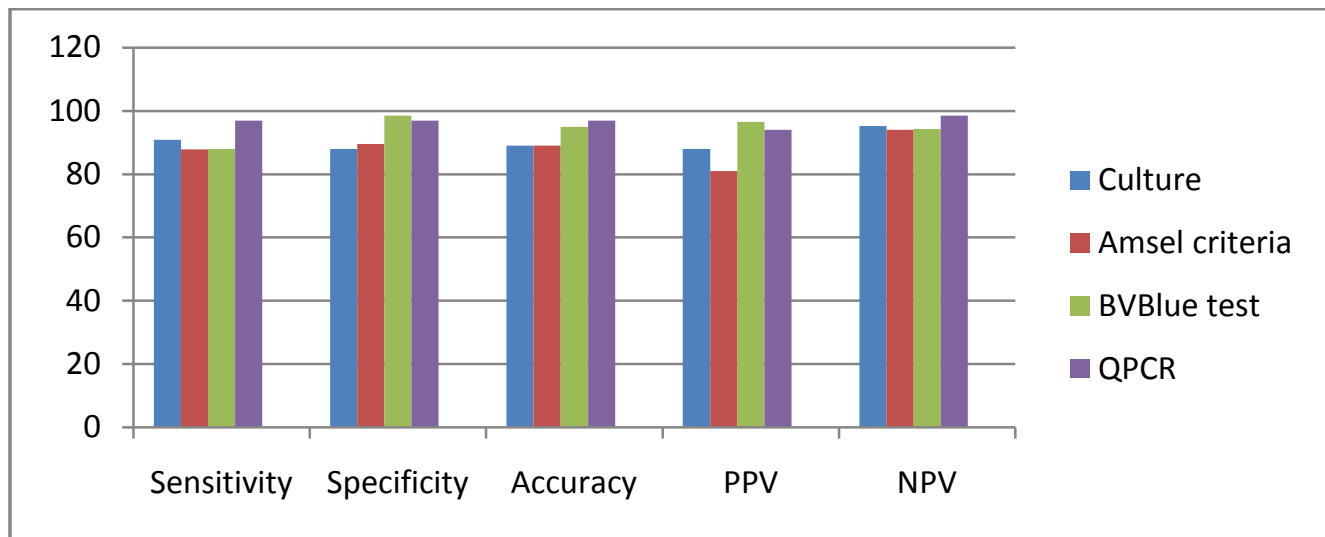

Figure (3): Percentages of sensitivity, specificity, accuracy, positive predictive value and negative predictive value of the different diagnostic methods.

\section{Discussion}

Bacterial vaginosis (BV) is an ecological disorder of the vaginal microbiota that affects millions of women annually, and is associated with numerous adverse health outcomes including pre-term birth and the acquisition of sexually transmitted infections. Due to these serious complications, there has been an increase of interest in bacterial vaginosis during the last decade [9], regarding its diagnosis and treatment. In this study, we recorded the prevalence of $\mathrm{BV}$ among population and determined the relation of the different risk factors and demographic profiles of patients with BV incidence and compared the different diagnostic methods of BV with Nugent score as a gold standard to evaluate them. We also also recorded the prevalence of BV among population and determined the relation of the different risk factors and demographic profiles of patients with $\mathrm{BV}$ incidence.

The results revealed that from the 100 patients, 33\% were diagnosed as bacterial vaginosis (by Nugent score), $23 \%$ were having candidiasis, $8 \%$ aerobic vaginitis and $3 \%$ trichomoniasis and $33 \%$ were normal. Different percentages of vaginal infection were reported by previous studies, some studies reported lower BV incidence rates such as Madhivanan et al., [24], who found that the prevalence of $\mathrm{BV}$ is $19 \%$ and trichomoniasis $8.5 \%$. And others reported higher BV incidence rates such as Lowe et al., [25], who reported that $42 \%$ of cases had bacterial vaginosis, $14 \%$ had candidiasis, $2 \%$ trichomoniasis, $16 \%$ mixed infections and $26 \%$ negative cases and Thulker et al., [26], who diagnosed cases of vaginitis as bacterial vaginosis (53.8\%), followed by mixed vaginitis (36.8\%), Candidiasis (6.2\%)and Trichomoniasis in (3.2\%).

The highest number of BV positive cases was in the age group of (26-35)Yrs, (18/33, 54.5\%). The (18-25) age group represented $18.2 \%$ of BV positive cases while the (36-45) age group represented $27.3 \%$ of $\mathrm{BV}$ positive cases. This means that BV incidence increased with increasing age and then decreased again. This agrees with previous studies like Thulkar, et al., [26], who reported that vaginal infections commonly occur in women of reproductive age i.e between 25-30 years. And Akhter et al., [21], who observed that the majority of the patients were within the age group of 26-35 Yrs (45.5\%) followed by 15 to 25 Yrs (41\%).

We observed that $94 \%$ of $\mathrm{BV}$ positive cases were married, this confirms the observation of the strong relation between sexual activity and BV acquisition reported by previous studies such as Verstraelen et al., [27], who reported that BV may be considered a sexually enhanced disease, with frequency of intercourse being a critical factor and Gallo et al., [28], who found a biological evidence of an association between semen exposure and incident BV. It was observed that $94 \%$ (31/33) of the BV positive cases were using vaginal douches which confirms that vaginal douches represent a risk factor of BV acquisition. Previous observational studies also suggested a strong association between vaginal douching and bacterial vaginosis [14, 29, 30]. 
BV positive cases using IUD were equal to BV positive cases using hormonal contraceptives including injection and tablets $(12 / 33,36.4 \%)$. This seems to mean that IUD didn't represent a risk factor for BV acquisition and hormonal contraceptives didn't represent a protective factor from BV acquisition. This observation agrees with Lessard et al., [31], who did not find any increase in risk for acquiring BV by using IUD. But disagrees with Donders et al., [32] \& Madden et al., [33] who have shown an increase in the risk of BV among IUD users. Also our finding disagrees with studies [26,34], that reported a protective effect of hormonal contraceptives against BV.

Regarding Nugent score as a method of BV diagnosis, $33 \%$ of cases were diagnosed to be BV positive. Lower percentages of BV prevalence using Nugent's method were reported by some studies [35, 36, 37, 38, 39], Which reported BV incidence rates of $7.1 \%, 19 \%, 24 \%, 23.03 \%$ and $19 \%$ respectively. Similar percentages were obtained by other studies [40, 41, 42], which reported BV prevalence rate as 32\%, 36.7\% \& $31.6 \%$ respectively. Higher percentage were obtained by Taj et al., [4], who found that BV incidence rate was $78 \%$. These differences in the incidence rates may be due to difference in the geographical distribution, hygienic measures and sexual habits between our research area and those studies' research areas.

Regarding culture, both $G$. vaginalis and A. vaginae were recovered from 38 (38\%) patients, so the number of BV positive cases were $38(38 \%)$. Lower percentage of BV detection by culture was achieved by Akhter et al., [21] and Pavani and Saileela, [38] who reported a BV incidence rate of $21 \%$ \& $15.91 \%$, respectively. In comparison with Nugent's criteria as a gold standard, this study found that culture had the following sensitivity, specificity, positive predictive value (PPV) and negative predictive value (NPV): $90.9 \%$, $88 \%, 79 \%$ and $95.2 \%$ respectively. These results are higher than those obtained by previous studies [36, 38], which reported for culture a sensitivity, specificity, positive predictive value (PPV) and negative predictive value (NPV) of $51 \%, 88.7 \%, 85.5 \%$ and $58 \%$ respectively and $42.55 \%, 92.99 \%, 64.51 \%$ and $84.39 \%$ respectively.

Regarding Amsel criteria, our study found that 36 (36\%) of the 100 cases, were BV positive as they met three or four of Amsel criteria. Lower rates of BV incidence were obtained by studies [38, 39, 43], in which BV was diagnosed $24 \%, 18 \%$ \& $24 \%$ of cases by Amsel's criteria. And higher rates of BV incidence were reported by other studies [4,42], which reported $62 \%$ \& 58\% BV incidence rate. In comparison with Nugent's criteria as a gold standard, we found that Amsel's criteria had $88 \%$ sensitivity, $89.6 \%$ specificity, $81 \%$ positive predictive value and $94 \%$ negative predictive value. Modak et al. [37], reported lower sensitivity $(66.67 \%)$, and similar specificity $(94.74 \%)$, positive predictive value $(80 \%)$ and negative predictive value (90\%). Taj et al., [4], reported a lower sensitivity of (77\%) and negative predictive value (53\%), similar specificity $(91 \%)$, and higher positive predictive value of $(97 \%)$. Khatoon et al., [44], obtained a lower sensitivity $(69 \%)$ and negative predictive value (72\%), similar specificity $(93.1 \%)$ and higher positive predictive value $(92.2 \%)$.

In this study, BV was diagnosed in 30 of the 100 patients (30\%) using BVBlue test. Lower rates $(22 \%, 21 \%)$ were observed by previous studies [21, 45 respectively] using BVblue test for BV diagnosis. Higher rate was observed by Khatoon et al., [44] who diagnosed BV in $60.8 \%$ of cases. In comparison with Nugent's criteria as a gold standard, BVBlue test had the following sensitivity, specificity, positive predictive value (PPV), negative predictive value (NPV) and accuracy: 88\%, 98.5\%, $96.6 \%, 94.3 \%$ and $95 \%$ respectively. Higher sensitivity was obtained by Akhter et al., [21] and Kampan et al., [14] who both found that BVBlue test had $100 \%$ sensitivity but they both obtained nearly the same specificity of ours ( $98 \% \& 98.3 \%$ respectively). Kampan et al., [14], reported a positive predictive value (PPV) of $94.4 \%$ and negative predictive value (NPV) of $100 \%$ compared to Gram stain and these are similar results to our study. Khatoon et al., [44], reported for BVBlue test a higher sensitivity $(95.3 \%)$ than ours and similar percentages of specificity $(92.1 \%)$, positive predictive value $(93.4 \%)$ and negative predictive value $(94.4 \%)$.

Using qPCR, The number of cases showing G. vaginalis DNA level $\geq 10^{9}$ copies $/ \mathrm{mL}$ and/or $A$. vaginae DNA level $\geq 10^{8}$ copies/mL were $34(34 \%)$ cases. According to this, $34(34 \%)$ cases were BV positive while $66(66 \%)$ cases were BV negative. Menard et al., [23], used the same concentration thresholds of $G$. vaginalis and A. vaginae and diagnosed BV in $21 \%$ of cases. Zozaya-Hinchliffe et al. [46], agree with us in that A. vaginae and G. vaginalis are present at higher concentrations in BV patients. Shipitsyna et al., [17], detected both $G$. vaginalis and A. vaginae by real-time PCR in $100 \%$ of the BV patients, but these bacteria were also detected in $78 \%$ and $63 \%$ of subjects without $\mathrm{BV}$, respectively, confirming that the qualitative detection of these two species had very low specificity and they should be detected quantitatively as we did. In comparison with Nugent score, this study showed that qPCR has $96.9 \%$ sensitivity, $97 \%$ specificity, $94 \%$ positive predictive value (PPV), $98.5 \%$ Negative predictive value (NPV) and $97 \%$ accuracy. These results agree with Menard et al., [23], who found that qPCR had similar sensitivity (100\%), specificity of 93\%, and negative predictive value of $100 \%$ but lower positive predictive value of $73 \%$ for predicting BV, and Shipitsyna et al., [17], who reported that the highest sensitivities and specificities were obtained when the depletion of Lactobacillus spp. was combined with the presence of either G. vaginalis or A. vaginae at 
diagnostic levels, as determined by qPCR (sensitivity $97 \%$, specificity $96 \%$ ). But we disagree with Catwright et al., [16], who observed that quantitative analysis of these 2 markers (G. vaginalis and A. vaginae) in combination could not reliably differentiate BV-positive from BV-negative individuals and that inclusion of markers with a higher degree of specificity, for example, BVAB-2 and Megasphaera-1, is necessary to produce an assay with adequate positive predictive value.

\section{Conclusion}

This study was done in upper Egypt and concluded that bacterial vaginosis (BV) incidence is between 30-38\% using the different diagnostic methods of BV. All the tested methods including Amsel criteria, culture, BVBlue $^{\circledR}$ test and quantitative PCR are reliable methods for diagnosis of BV when compared with Nugent score as a gold standard. Culture, Amsel criteria \& BVBlue ${ }^{\circledR}$ test had substantial agreement with the gold standard while qPCR had almost perfect agreement with the gold standard. However, these different methods have different values of sensitivity, specificity, positive predictive value (PPV), negative predictive value (NPV) and accuracy. QPCR was the most sensitive and accurate method while BVBlue ${ }^{\circledR}$ test was the most specific one.

\section{References}

[1]. Z. Ling, J. Kong, F. Liu, H. Zhu, X. Chen, Y. Wang, L. Li, K.E. Nelson, Y. Xia, and C. Xiang, Molecular analysis of the diversity of vaginal microbiota associated with bacterial vaginosis, BMC Genomics, 11, 2010, 488-504.

[2]. C.H. Livengood, Bacterial vaginosis: an overview. Rev. Obstet \& Gynecol., 2 (1), 2009, 28-37.

[3]. P. Nyirjesy, Vulvovaginal candidiasis and bacterial vaginosis. Infect Dis Clin North Am., 22, 2008, 637-652.

[4]. Y. Taj, D. Nasir, N. Kahkashan, and A. Anjum, Sensitivity and specificity of rapid clinical diagnostic test for bacterial vaginosis and its analytical value, J Dow Uni Health Sci., 6(3), 2012, 91-94.

[5]. J.P. Menard, F. Fenollar, D. Raoult, L. Boubli, and F. Bretelle, Self-collected vaginal swabs or the quantitative real-time Polymerase Chain reaction assay of Atopobium vaginae and Gardnerella vaginalis and the diagnosis of bacterial vaginosis, Eur J Clin Microbiol Infect Dis., 31, 2012, 513-518.

[6]. T.L. Cherpes, S.L. Hillier, L.A. Meyn, J.L. Busch, and M.A. Krohn, A delicate balance: risk factors for acquisition of bacterial vaginosis include sexual activity, absence of hydrogen peroxide-producing lactobacilli, black race, and positive herpes simplex virus type 2 serology. Sex Transm Dis, 35, 2008, 78-83.

[7]. R.M. Brotman, M.A. Klebanoff, T.R. Nansel, K.F. Yu, W.W. Andrews, J. Zhang, and J.R. Schwebke, Bacterial vaginosis assessed by gram stain and diminished colonization resistance to incident gonococcal, chlamydial, and trichomonal genital infection. J Infect Dis. 202(12), 2010, 1907- 1915.

[8]. E. Gillet, J.F.A. Meys, H. Verstraelen, C. Bosire, P. Philippe De Sutter, M. Temmerman, and D.V. Broeck, Bacterial vaginosis is associated with uterine cervical human papillomavirus infection: a meta-analysis, BMC Infect Dis, 11, 2011, 1-9.

[9]. K. Yoshimura, N. Morotomi, K. Fukuda, M. Nakano, H.T. Kashimura and H. Taniguchi, Intravaginal microbial flora by the 16S rRNA gene sequencing, Am J Obstet Gynecol. 205, 2011, 235.e1-9.

[10]. R. Amsel, P.A. Totten, C.A. Spiegel, K.C. Chen, D. Eschenbach, and K.K. Holmes, Nonspecific vaginitis. Diagnostic criteria and microbial and epidemiologic associations. Am J Med. 74, 1983, 14-22.

[11]. R.P. Nugent, M.A. Krohn, and S.L. Hillier, Reliability of diagnosing bacterial vaginosis is improved by a standardized method of Gram stain interpretation, J. Clin. Microbiol. 29, 1991, 297-301.

[12]. S. Mohanty, S. Sood, A. Kapil, and S. Mittal, Interobserver variation in the interpretation of Nugent scoring method for diagnosis of bacterial vaginosis, Indian J Med Res., 131, 2010, 88-91.

[13]. C.S. Bradshaw, S.N. Tabrizi, C.K. Fairley, A.N. Morton, E.A. Rudland, and S.M. Garland, Association of Atopobium vaginae and Gardnerella vaginalis with Bacterial Vaginosis and Recurrence after Oral Metronidazole Therapy. J Infect Dis. 194(6), 2006, 828-836.

[14]. N.C. Kampan, S.S. Suffian, N.S. Ithnin, M. Muhammad, S.Z. Zakaria, and M.A. Jamil, Evaluation of BV® Blue Test Kit for the diagnosis of bacterial vaginosis. J Sex Reprod Healthc., 2, 2011, 1-5.

[15]. D.N. Fredricks, T.L. Fiedler, and J.M. Marrazzo, Moleculal identification of bacteria associated with bacterial vaginosis, $N$ Eng J Med, 353, 2005, 1899-1911.

[16]. C.P. Catwright, B.D. Lembke, K. Ramachandran, B.A. Body, M.B. Nye, C.A. Rivers, and J.R. Schwebke, Development and validation of a semiquantitative, multitarget PCR assay for diagnosis of bacterial vaginosis. J Clin Microbiol. 50(7), 2012, 23212329 .

[17]. E. Shipitsyna, A. Roos, R. Datcu, A. Halle'n, H. Fredlund, J.S. Jensen, L. Engstrand, and M. Unemo, Composition of the vaginal microbiota in women of reproductive age-sensitive and specific molecular diagnosis of bacterial vaginosis is possible ? PLoS ONE . 8 (4), 2013, e60670.

[18]. P.A. Totten, R. Amsel, J. Hale, P. Piot, and K.K. Holmes, Selective differential human blood bilayer media for isolation of Gardnerella (Haemophilus) vaginalis, J. Clin. Microbiol. 15, 1982, 141-147.

[19]. M.D. Harwich, J.M. Alves, G.A. Buck, J.F. Strauss, J.L. Patterson, A.T. Oki, P.H. Girerd, and K.K. Jefferson, Drawing the line between commensal and pathogenic Gardnerella vaginalis through genome analysis and virulence studies. BMC Genomics 11, 2010, 375 .

[20]. J.P. Burton, C.N. Chilcott, M. Al-Qumber, H.J. Brooks, D. Wilson, J.R. Tagg, and C. Devenish, A preliminary survey of Atopobium vaginae in women attending the Dunedin gynaecology out-patients clinic: is the contribution of the hard-toculture microbiota overlooked in gynaecological disorders? Aust N Z J Obstet Gynaecol. 45(5), 2005, 450-452.

[21]. S. Akhter, H. Satter, S. Tarafder, R.A. Miah, S. Sharmin, and S. Ahmed, Rapid Detection of Bacterial Vaginosis (BV) by BVBlue test, Bangladesh J Med Microbiol., 4 (1), 2010, $24-27$.

[22]. M.R. Zariffard, M. Saifuddin, B.E. Sha, and G.T. Spear, Detection of bacteria vaginosis-related organisms by real-time PCR for lactobacilli, Gardnerella vaginalis and Mycoplasma hominis, FEMS Immunol. Med. Microbiol., 34, 2002, 277-281.

[23]. J.P. Menard, C. Mazouni, F. Fenollar, D. Raoult, L. Boubli, and F. Bretelle, Diagnostic Accuracy of Quantitative Real-Time PCR assay versus Clinical and Gram Stain Identification of Bacterial Vaginosis, Eur J Clin Microbiol Infect Dis., 29 (12), 2010, $1547-1552$. 
[24]. P. Madhivanan, K. Krupp, J. Hardin, C. Karat, J.D. Klausner, and A.L. Reingold, Simple and inexpensive point-of-care tests improve diagnosis of vaginal infections in resource constrained settings, Trop Med Int Health., 14(6), $2009,703-708$.

[25]. N.K. Lowe, J.L. Neal, and N.A. Ryan-Wenger, Accuracy of the clinical diagnosis of vaginitis compared to a DNA Probe Laboratory Standard, Obstet Gynecol., 113(1), 2009, 89-95.

[26]. J. Thulkar, A. Kriplani, N. Agarwal, and S. Vishnubhatla, Aetiology \& risk factors of recurrent vaginitis \& its association with various contraceptive methods. Indian J Med Res., 131, 2010, 83-87.

[27]. H. Verstraelen, R. Verhelst, M. Vaneechoutte, and M. Temmerman, The epidemiology of bacterial vaginosis in relation to sexual behaviour. BMC Infect Dis. 10, $2010,81$.

[28]. M.F. Gallo, L. Warner, C.C. King, J.D. Sobel, R.S. Klein, S. Cu-Uvin, A.M. Rompalo, and D.J. Jamieson, Association between Semen Exposure and Incident Bacterial Vaginosis. Infect Dis Obstet Gynecol., 2011, 842652.

[29]. S. Srinivasan, C. Liu, C.M. Mitchell, T.L. Fiedler, K.K. Thomas, K.J. Agnew, J.M. Marrazzo, D.M. Fredricks, Temporal variability of human vaginal bacteria and relationship with bacterial vaginosis, PLOS ONE, 5 (4), $2010, \mathrm{e} 10197$.

[30]. J. Mangot-Bertrand, F. Fenollar, F. Bretelle, M. Gamerre, R. Didier, and B. Courbierem, Molecular diagnosis of bacterial vaginosis: impact on IVF outcome. Eur J Clin Microbiol Infect Dis., 32 (4), 2013, 535-541.

[31]. T. Lessard, J.A. Simoes, M.G. Discacciati, M. Hidalgo, and L. Bahamonde Cytological evaluation and investigation of the vaginal flora long-term users of the levonorgestrel-releasing intrauterine system (LNG-IUS), Contraception, 77, 2008, 30 -33 .

[32]. G.G. Donders, J. Berger, H. Heuninckx, G. Bellen, and A. Comelis, Vaginal flora changes on Pap smears after insertion of levonorgestrel releasing intrauterine device. Contraception, 83, 2011, 352-356.

[33]. T. Madden, J.M. Grentzer, G.M. Secura, J.E. Allsworth, and J.F. Peipert, Risk of bacterial vaginosis in users of the intrauterine device: A longitudinal study, Sex Transm Dis., 39 (3), 2012, 217-222.

[34]. M. Riggs, M. Klebanoff, T. Nansel, J. Zhang, J. Schwebke, and W. Andrews Longitudinal association between hormonal contraceptives and bacterial vaginosis in women of reproductive age, Sex Transm Dis., 34, 2007, 954-959.

[35]. T.N.D. Alice, S. Kives, L. Merovtiz, R. Nitsch, K. Teessler, and M.H. Yudin, Screening for Bacterial Vaginosis at the Time of Intrauterine Contraceptive Device Insertion: Is There a Role?, J Obstet Gynaecol Can, 34(2), 2012, $179-185$.

[36]. R. Mathew, R. Sudhakshina, M. Kalyani, S. Jayakumar, B. Lal, and S. Banu, Microbiological profile of vaginosis among women of the reproductive age group, who attended a tertiary care hospital, J Clin Diag Res., 5(8), $2011,1548-1551$.

[37]. T. Modak, P. Arora, C. Agnes, R. Ray, S. Goswami,P. Ghosh, P. and N.K. Das, Diagnosis of bacterial vaginosis in cases of abnormal vaginal discharge: comparison of clinical and microbiological criteria, J Infect Dev Ctries., 5(5), 2011, 353-360.

[38]. K. Pavani, and K. Saileela, Diagnosis of bacterial vaginosis in reproductive age group in tertiary health care hospital in south India: Comparison of clinical and microbiological criteria, J Evolut Med Dent Sci., 2(35), 2013, 6611-6615.

[39]. B. Udayalaxmi, G. Bhat, S. Kotigadde, and S. Shenoy, Comparison of the Methods of Diagnosis of Bacterial Vaginosis, $J$ Clin Diag Res., 5(3), 2011, 498-501.

[40]. S. Ayenalem, L. Yusuf, and M. Ashenafi, Lactic Acid Bacterial vaginosis among Outpatients in Addis Ababa. Ethiop. J. Health Dev. 24(3), 2010, 198-204.

[41]. V. Chaudhary, V. Prakesh, K. Agarwal, V.K. Agrawal, A. Singh, and S. Pandey, Clinico-Microbiological profile of women with vaginal discharge in a tertiary care hospital of northern India, Intern J Med Science and Public Health. 1(2), 2012, 75-80.

[42]. A.A. Rangari, S. Parmjit and V.K. Sharma, Comparison of the Amsel's composite clinical criteria and Nugent's criteria for diagnosis of bacterial vaginosis : A step towards preventing misdiagnosis, JARBS. Biol Sciences., 5(1), $2013,37-44$.

[43]. N. Begum, N. Muazzam, S.M. Shamsuzzaman, A. Chowdhury, A. Rashid, and D. Islam, Diagnosis of Bacterial Vaginosis by Acridine Orange Staining and its Comparison to Conventional Methods and Association of vaginalis with Bacterial Vaginosis. Bangladesh J Med Microbiol. 4 (01), 2010, 37- 42.

[44]. R. Khatoon, N. Jahan, S. Ahmed, and T. Rabbani, Comparison of OSOM BV Blue test with conventional methods for diagnosis of bacterial vaginosis. Afr. J. Microbiol. Res. 7(28), 2013, 3698-3703.

[45]. L. Myziuk, B. Romanowski, and S.C. Johnson, BVBlue test for diagnosis of bacterial vaginosis, J Clin Microbiol., 41(5), 2003, $1925-1928$.

[46]. M. Zozaya-Hinchliffe, R. Lillis, D.H. Martin, and M.J. Ferris, Quantitative PCR assessments of Bacterial Species in Women with and without Bacterial Vaginosis, J Clin Microbiol., 48(5), 2010, 1812-1819. 